\title{
LINK BETWEEN HIPPARCOS AND VLBI CELESTIAL REFERENCE FRAMES
}

\author{
E.F.Arias. (Facultad de Cs. Astronomicas y Geofisicas de La Plata, CONICET, \\ Central Bureau of IERS) \\ J.-F. Lestrade (Observatoire de Paris, Central Bureau of IERS)
}

\begin{abstract}
The celestial frame of HIPPARCOS astrometric satellite will be materialized by the positions and proper motions of about 120000 stars relative to arbitrary origins. As the HIPPARCOS reference frame is not naturally related to fixed directions in space, it has to be linked to an inertial frame of similar quality. The technique of VLBI determines the coordinates of extragalactic radio sources precise at the milliarcsecond level in an equatorial frame. The precision expected for HIPPARCOS normal mission is 0.002 " for the positions, yearly proper motions and parallaxes.
\end{abstract}

\section{Introduction}

The link between the VLBI and HIPPARCOS reference frames will a) "stop" the rotation of reference frame produced by the satellite, b) densify the extragalactic reference frame in optical frequencies, $c$ ) unify the radio and optical coordinate systems and allow direct comparison of the images of celestial objects obtained with radio and optical techniques with the same angular resolution.

Radio stars provide a direct link since they can be observed with both techniques without intermediate objects. To assure good observational conditions by the satellite, they must be brighter than $m=11$. Their flux densities must be at least of some $m J y$ to be adapted to the VLBI sensitivity. Besides that, their optical and radio images must be coincident at $0.002 "$. Following these criteria Lestrade et al. (1982) have established a list of 22 radio stars candidate to the link. Most of the objects in this list are close binaries RSCVn with angular separations < 0.004" and flux densities < $50 \mathrm{mJy}$.

\section{The method}

We have studied the link between HIPPARCOS and VLBI frames on the basis of observations of radio stars.

In absence of regional deformations, the link between two frames with the same origin can be mathematically expressed by a simple rotation. If the relative orientation of the frames evolves linearly with time, the link is given by a global rotation at an arbitary epoch, represented by a matrix $[R]$ and by a matrix $[\dot{R}]$ which represents the angular velocity of rotation of one frame relative to the other.

For each radio star $i$, the technique of VLBI provides a vector $\vec{\sigma}_{\mathrm{Vi}}$ at an epoch $t_{\mathrm{Vi}}$ of observation of the star, and an associated proper motion $\dot{\sigma}_{\mathrm{Vi}}$. On the other hand, the observation of the satellite will provide vector $\vec{\sigma}_{\mathrm{Hi}}$ at an epoch $\mathrm{t}_{\mathrm{Hi}}$ of observation and an associated proper motion $\dot{\vec{\sigma}}_{\mathrm{Hi}}$. 
At an arbitrary instant $t$, the link equations between both frames given by one commonly observed radio star are:

$$
\begin{aligned}
& \overrightarrow{\sigma_{V i}(t)}=[R(t)] \cdot \overrightarrow{\sigma_{H i}}(t) \\
& \left.\dot{\overrightarrow{\sigma_{V i}}}=[R(t)] \cdot \dot{\overrightarrow{\sigma_{H i}}}+[\dot{R}] \cdot \overrightarrow{\sigma_{H i}}(t)\right)
\end{aligned}
$$

The relative orientation between the two frames at an initial epoch $t_{0}$ is represented by three rotations $A_{1}, A_{2}$, and $A_{3}$ around the axes of one frame, and the angular velocity of rotation is represented by the time - derivatives $\dot{A}_{1}, \dot{A}_{2}$ and $\dot{A}_{3}$. Expressions (1) and (2) provide four independent equations in the coordinates. Two radio stars are enough to evaluate the unknowns; however we will consider a greater number of objects to perform a least squares adjustment.

We have adopted in this analysis the equatorial coordinate system because it is the natural system of the extragalactic VLBI frame. The FAST consortium has adopted the ecliptic coordinate system to reduce HIPPARCOS data. Equations (1) and (2) represent the link between the ecliptic coordinate system of HIPPARCOS and the VLBI equatorial coordinate system. It will be more expeditive to transform the ecliptic coordinates of HIPPARCOS observation into equatorial coordinates system before realising the link. In these conditions, the angles $A_{1}, A_{2}$, and $A_{3}$ will be small quantities at the level of $10^{-7}$ rad and the matrices, to the first order in the angles and in the velocity components, can be written:

$$
\left[R\left(t_{0}\right)\right]=\left[\begin{array}{ccc}
1 & A_{3} & -A_{2} \\
-A_{3} & 1 & A_{1} \\
A_{2} & -A_{1} & 1
\end{array}\right] \quad[\dot{R}] \quad=\left[\begin{array}{ccc}
0 & \dot{A}_{3} & -\dot{A}_{2} \\
-\dot{A}_{3} & 0 & \dot{A}_{1} \\
\dot{A}_{2}-\dot{A}_{1} & 0
\end{array}\right]
$$

\section{The radio stars selected for the link}

14 radio stars with $\mathrm{mv}<11$ have been used for the link. Their distribution in the sky is shown in figure 1. Their VLBI coordinates at J2000.0, their proper motions and the corresponding epochs of observation are in Table 1. Data of the eight stars indicated with (1) had been obtained with the MarkIII data acquisition system and analysed in the MASTERFIT by Lestrade et al. (1988). The four objects at negative declinations indicated with (2) have been observed by White $e$ t al. (1990). The positions of the two stars indicated with (3) in Table 1 were also provided by Lestrade and have been determined with the VLA. All the positions are in J2000.0 by means of the standard precession-nutation models IAU 1976/1980.

Figure 1.

Radio stars considered.

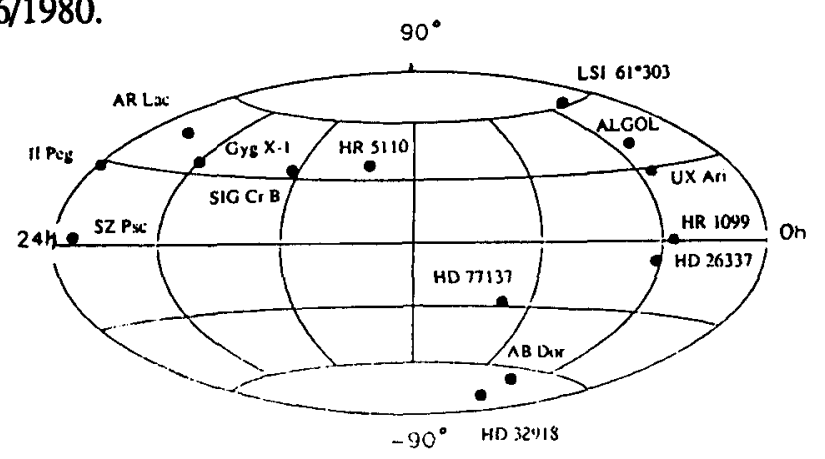


Table 1: VLBI positions and proper motions of the radio stars

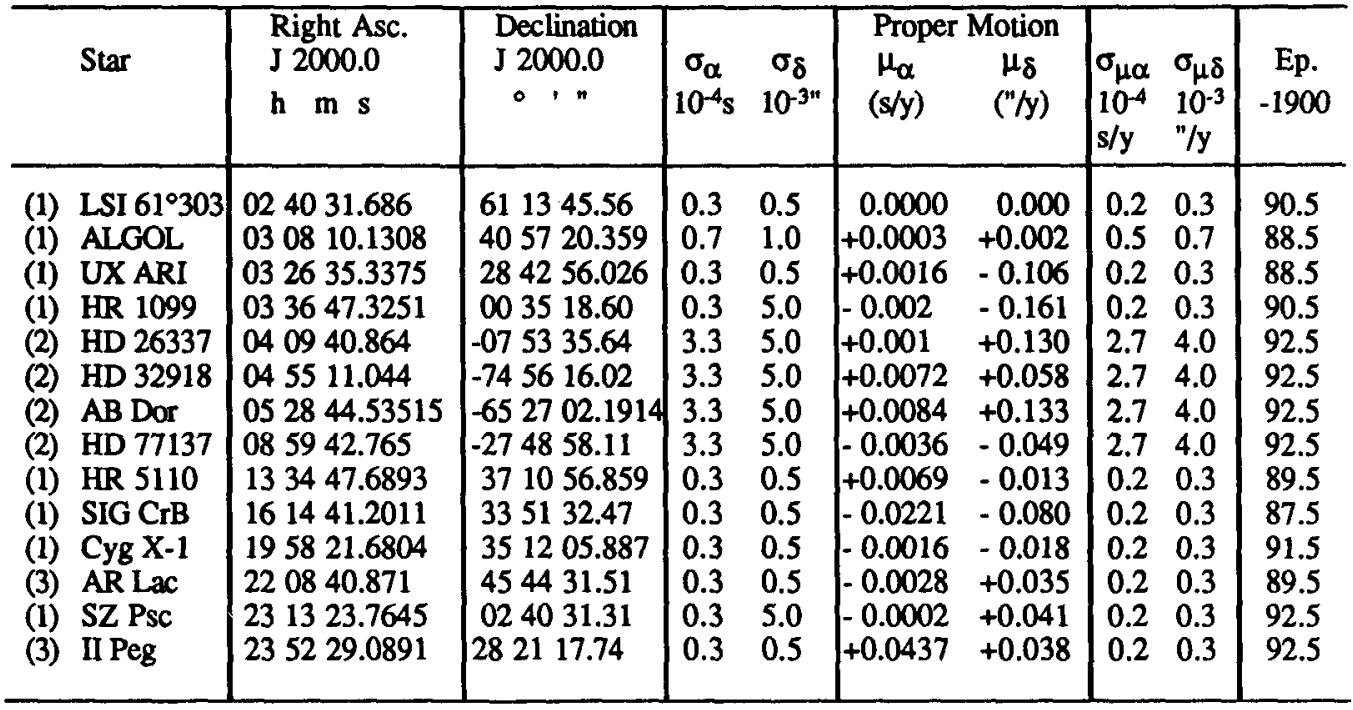

\section{The simulation of HIPPARCOS observations}

We have simulated the positions and proper motions $\vec{\sigma}_{\mathrm{Hi}}\left(\mathrm{t}_{\mathrm{Hi}}\right), \dot{\vec{\sigma}}_{\mathrm{Hi}}$ for each radio star "observed" with HIPPARCOS from the known VLBI vectors $\sigma_{\mathrm{Vi}}\left(t_{\mathrm{Vi}}\right), \sigma_{\mathrm{Vi}}$ given by Lestrade et al. (1988). The relative orientation between the two frames at the arbitrary epoch $t_{0}=1991.0$ has been fixed by adopting the values

$$
A_{1}=+0.030^{\prime \prime}, A_{2}=+0.025^{\prime \prime}, A_{3}=-0.045^{\prime \prime} \text {. }
$$

The time evolution of the frames from the epoch $t_{0}$ is given by

$\dot{A}_{1}=+0.002 " /$ year, $\dot{A}_{2}=-0.003 " /$ year, $\dot{A}_{3}=+0.001 " /$ year.

The equations (1) and (2) for $t=t_{H i}$ have been used to simulate HIPPARCOS observations.

To make the simulation realistic, we have added gaussian noise to the VLBI and HIPPARCOS positions and proper motions. The uncertainties are also realistic; for HIPPARCOS data they are those expected for the mission, and for the VLBI observations, they have been assigned according to the caracteristics of the networks. 


\section{Results}

We have analysed the quality of the link in two cases, considering either all the stars or only objects in the Northern hemisphere.

First, we have simulated HIPPARCOS as described in the previous section. Then, we have reevaluated $\mathrm{A}_{\mathrm{k}}, \dot{A}_{\mathrm{k}}(\mathrm{k}=1,3)$ from the vectors $\vec{\sigma}_{\mathrm{VP}} \dot{\vec{\sigma}}_{\mathrm{Vj}}$ and the simulated $\overrightarrow{\sigma_{\mathrm{HP}}} \dot{\overrightarrow{\sigma_{\mathrm{Hi}}}}$. The results of the two cases of link analysed are shown in Table 2.

The relative orientation at the initial epoch $\left(A_{k}\right)$ is determined with a precision at the level of $0.001 "$ ". The precision of the components $\dot{A}_{k}$ of the angular velocity is in the range $0.0007^{\prime \prime}-0.0009^{\prime \prime}$.

Concerning the distribution of objects, the inclusion of radio stars at negative declinations, with uncertainties several times greater than those in the North, does not improve the link and introduces a bias in $A_{1}, A_{2}$ and $A_{3}$.

Table 2: Results of the link between HIPPARCOS and VLBI reference frames via radio stars. Units of $A_{k}=0.001 "$, units of $\dot{A}_{k}$ : $0.001 " /$ year. $\sigma$ are the post-fit residuals, in units of $0.001 ", R$ is the goodness-of-fit parameter.

\begin{tabular}{l|c|c}
\hline \multicolumn{1}{c|}{$\mathrm{N}$} & All stars & $\begin{array}{c}10 \\
\text { Northern Stars }\end{array}$ \\
\hline $\mathrm{A} 1$ & $+31.09 \pm 0.91$ & $+31.45 \pm 0.87$ \\
$\mathrm{~A} 2$ & $+23.23 \pm 0.86$ & $+23.27 \pm 0.79$ \\
$\mathrm{~A} 3$ & $-44.93 \pm 0.73$ & $-44.65 \pm 0.71$ \\
& & \\
$\dot{\mathrm{A}} 1$ & $+1.49 \pm 0.82$ & $+1.44 \pm 0.79$ \\
$\dot{\mathrm{A}} 2$ & $-2.78 \pm 0.77$ & $-2.98 \pm 0.72$ \\
$\dot{\mathrm{A}} 3$ & $+1.08 \pm 0.67$ & $+1.55 \pm 0.66$ \\
$\sigma$ & 1.92 & 1.55 \\
$\mathrm{R}$ & 1.55 & 1.25 \\
\hline
\end{tabular}

\section{REFERENCES}

Lestrade, J.-F., Preston, R.A., Slade, M.A. 1982: Very Long Baseline Interferometry techniques, F. Biraud (ed.), CNES, p. 199.

Lestrade, J.-F., Requième, J., Rapapport, M., Preston, R.A. 1988: in The Earth's Rotation and Reference Frames for Geodesy and Geodynamics, G.W. Wilkins and A. Babcock (eds.), p. 67.

White, G.W., Lestrade, J.-F., Jauncey, D.L., Philips, R.B., Preston, R.A., Reynolds, J.E. 1990: Astron. J., 99, 1005. 